\title{
Philosophiques
}

\section{Référence et dénotation des termes scientifiques}

\section{Éric Bourneuf}

Volume 18, numéro 2, automne 1991

URI : https://id.erudit.org/iderudit/027151ar

DOI : https://doi.org/10.7202/027151ar

Aller au sommaire du numéro

Éditeur(s)

Société de philosophie du Québec

ISSN

0316-2923 (imprimé)

1492-1391 (numérique)

Découvrir la revue

Citer cet article

Bourneuf, É. (1991). Référence et dénotation des termes scientifiques. Philosophiques, 18(2), 27-62. https://doi.org/10.7202/027151ar

\section{Résumé de l'article}

Le point de départ de l'article est la théorie de la signification et de la référence des termes scientifiques présentée par Hilary Putnam dans son article « The Meaning of 'Meaning7 » et quelques autres essais de Mind, Language and Reality. Dans la partie critique du texte la thèse et les arguments de Putnam, ainsi que sa prétention d'éviter le problème de rincommensurabilité des théories rivales, sont évalués à la lumière de la distinction que nous introduisons entre référence et dénotation. Dans sa partie constructive le texte propose les grandes lignes d'un traitement de la référence (et de la dénotation) des termes scientifiques qui s'appuie sur notre conception de la structure de la théorie physique, le pragmatisme constructif. 


\title{
RÉFÉRENCE ET DÉNOTATION DES TERMES SCIENTIFIQUES
}

\author{
par Éric Bourneuf
}

RÉsumé. Le point de départ de l'article est la théorie de la signification et de la référence des termes scientifiques présentée par Hilary Putnam dans son article "The Meaning of 'Meaning' " et quelques autres essais de Mind, Language and Reality. Dans la partie critique du texte la thèse et les arguments de Putnam, ainsi que sa prétention d'éviter le problème de l'incommensurabilité des théories rivales, sont évalués à la lumière de la distinction que nous introduisons entre référence et dénotation. Dans sa partie constructive le texte propose les grandes lignes d'un traitement de la référence (et de la dénotation) des termes scientifiques qui s'appuie sur notre conception de la structure de la théorie physique, le pragmatisme constructif.

ABSTRACT. The paper's starting point is Hilary Putnam's theory of meaning and reference for scientific terms, as presented in "The Meaning of 'Meaning' " and several other essays from his Mind, Language and Reality. A critique of Putnam's thesis and arguments, as well as of his claim to avoid the problem of incommensurability of competing theories, is offered in the light of our own distinction between reference and denotation. In the process we develop an outline of a theory of reference (and denotation) for scientific terms that builds on our view of the structure of physical theory: constructive pragmatism. 


\section{INTRODUCTION}

Notre but principal dans cet article est d'exposer les grandes lignes $d$ 'une théorie de la référence pour les termes d'espèces naturelles (ex.: quark) et de grandeurs physiques (ex.: charge électrique). Nous avons choisi comme point de départ la théorie que présente Hilary Putnam dans son article «The Meaning of 'Meaning'" (et quelques autres textes de Mind, Language and Reality) parce qu'elle favorise l'entrée en matière de notre sujet. Cette théorie est en fait plus généralement une théorie de la signification qui intègre des éléments d'une théorie de la compréhension du langage et une théorie de la référence. Une des raisons ayant motivé son élaboration est la popularité, à l'époque, des discussions sur l'incommensurabilité des théories physiques rivales, qui, selon des auteurs comme Kuhn et Feyerabend, semblait suivre de considérations portant sur la signification des termes théoriques. En opposition avec ces conclusions, Putnam cherchait une théorie de la signification permettant de préserver les notions de référence et de vérité extra-théoriques nécessaires pour la comparaison des théories et, ultimement, pour expliquer le progrès dans les sciences. Par rapport à ce groupe de problèmes nous nous sommes fixé deux buts intermédiaires, qui vont nous permettre de rejoindre peu à peu notre objectif principal: nous voulons tout d'abord montrer que la théorie des termes d'espèces naturelles et de grandeurs physiques (ci-après nommés simplement termes "scientifiques") présentée dans "The Meaning of 'Meaning' » est inadéquate à la fois du point de vue de la référence et de la signification, et ensuite que la théorie de la signification traditionnelle que critique Putnam est valable sur le plan sémantique sans que cela entraîne pour autant les problèmes que l'on croit.

Le plan de cet article est le suivant: nous rappellerons premièrement les traits principaux de la théorie de la signification traditionnelle et la façon dont elle semble impliquer que la référence est relative à chaque théorie, entraînant ainsi le problème de l'incommensurabilité. Nous décrirons ensuite la théorie de Putnam, les arguments en sa faveur, de même que la manière dont elle prétend résoudre les difficultés de sa rivale. Trois exemples tirés de l'histoire de la physique serviront alors à mettre en évidence autant de lacunes dans la théorie de Putnam, comme théorie causale, comme théorie de la signification et comme théorie de la référence. Cette partie de la critique semblera 
nous ramener en arrière vers la conception traditionnelle. Ayant introduit la distinction entre la relation de dénotation (qui prévaut entre les expressions linguistiques et les objets) et celle de référence (que nous réserverons alors pour caractériser le lien entre des locuteurs et des objets), nous préciserons ce que nous voulons retenir de la théorie traditionnelle sur le plan sémantique. Le terrain sera alors préparé pour illustrer comment les discussions sur l'incommensurabilité dissimulent cette confusion entre la relation de dénotation et celle de référence, en plus de présupposer une conception erronée de la nature des théories, qu'elles retiennent de l'empirisme logique. En conclusion nous décrirons les principaux types de référence présents en sciences et donnerons quelques éléments d'une théorie de la dénotation des termes scientifiques qui s'appuie sur notre conception de la structure des théories physiques, le pragmatisme constructif ${ }^{1}$, qui veut éviter les faux pas de l'empirisme logique.

\section{L'ARGUMENT MENANT À L'INCOMMENSURABILITÉ}

L'incommensurabilité des théories physiques rivales est un problème aux multiples facettes - ce texte ne saurait donc en faire un examen détaillé - que nous ne voulons aborder, à la suite de Putnam dans les textes cités, que du point de vue de la référence. L'argument en faveur de la thèse qu'il attribue à Kuhn et Feyerabend (1978, p. 22) et perçoit comme une menace à sa position réaliste, résulte de la conjonction de deux thèses intuitivement très plausibles ${ }^{2}$. La première est la conception holiste de la signification qui prétend que la signification des termes théoriques est spécifiée par leur rôle à l'intérieur de chaque théorie. On se rappelle que le modèle standard de l'empirisme logique séparait le langage scientifique en une partie observationnelle et une partie théorique. Les termes du premier vocabulaire pouvaient être définis par leur connexion directe avec l'expérience, mais ce n'était pas le cas des termes théoriques. Après de nombreuses tentatives infructueuses pour contrer la difficulté on dut se résoudre à accepter une forme de la conception holiste. Pis encore, avec le rejet de la distinction entre le langage théorique et le langage observationnel, le holisme semblait s'étendre à tous les termes de la théorie ${ }^{3}$. Chaque changement de théorie devait donc impliquer un changement dans la signification de tous les termes considérés. La seconde thèse conduisant au problème de l'incommensurabilité est celle que Putnam appelle la conception traditionnelle de la signification, 
qui remonte à Frege. Deux idées la sous-tendent: (1) comprendre la signification d'un terme $c^{\prime}$ est être dans un état psychologique en vertu duquel se produit la saisie de son intension (qui est quelque chose comme l'ensemble de propriétés qui y est associé); (2) l'intension d'un terme fournit une condition nécessaire et suffisante pour en déterminer l'extension, c'est-à-dire l'ensemble des objets qui le satisfont. L'intension associée à " célibataire", par exemple, contient les propriétés "adulte " et "non marié "; un objet fera donc partie de l'extension de " célibataire " si et seulement si il est à la fois adulte et non marié. Pour voir que l'incommensurabilité suit de cette série de thèses il suffit de les mettre bout à bout:

(I) la signification d'un terme est déterminée par son intension;

(2) l'intension des termes scientifiques varie en fonction de la théorie dans laquelle ils sont définis;

(3) le même terme dans différentes théories n'a pas nécessairement la même intension;

(4) l'intension d'un terme en détermine l'extension;

(5) la notion d'extension (de référence) est intra-théorique;

(6) il est possible que des théories différentes utilisant les mêmes termes - et donc en apparence rivales - ne peuvent en fait pas être comparées car elles ne réfèrent pas au même domaine d'objets.

La conclusion (6) est la version de la thèse d'incommensurabilité à laquelle Putnam s'oppose:

To a strongly antirealistic intuition it makes little sense to say that what is in the extension of Archimedes' term "gold" is to be determined using our theory. For the antirealist does not see our theory and Archimedes' theory as two approximately correct descriptions of some fixed realm of theory-independant entities, and he tends to be skeptical about the idea of " convergence " in science - he does not think our theory is a better description of the same entities that Archimedes was describing. [...] The antirealist can use truth intra-theoretically in the sense of a "redundancy theory "; but he does not have the notions of truth and reference available extra-theoretically. $(1975$, p. 236)

\section{LA RÉPONSE DE PUTNAM}

La stratégie employée par Putnam pour faire face à ce problème l'amène à s'opposer aux deux thèses que nous avons exposées dans la section précédente. En résumé, Putnam va maintenir que l'extension d'un terme scientifique est déterminée en partie indexicalement et en partie socialement, mais pas, 
comme c'est le cas dans la théorie traditionnelle, par son intension. Putnam va également affirmer que, contrairement aux prétentions de la théorie holiste, c'est la référence (ou l'extension), et non pas la théorie physique, qui détermine en bonne partie la signification des termes scientifiques.

Putnam offre deux types d'arguments pour montrer que l'état psychologique d'un locuteur, par l'intermédiaire de l'intension qu'il associe à un terme scientifique, ne peut suffire à fixer son extension (la conjonction des deux thèses de la théorie traditionnelle). Un premier argument nous met devant des situations hypothétiques et nous demande d'évaluer nos intuitions sémantiques. L'histoire de la Terre-Jumelle est l'exemple le plus connu de ce type d'argument. Imaginons qu'il y a sur une planète jumelle de la Terre des lacs et des rivières remplis $d^{\prime}$ un liquide en apparence identique à l'eau à tout point de vue, mais dont la composition chimique est en fait $X Y Z$ (une abréviation pour une formule complexe) et non pas $\mathrm{H}_{2} \mathrm{O}$. Qui plus est, les habitants de la Terre et de la Terre-Jumelle parlent la même langue et utilisent tous deux le mot "eau" pour désigner ces liquides. Reportons-nous maintenant à une période de l'histoire où les habitants des deux planètes n'ont pas les connaissances scientifiques requises pour distinguer chimiquement les deux liquides. Dans ce contexte l'intension du mot "eau", et donc les états psychologiques des locuteurs qui l'utilisent, devaient être les mêmes dans tous les cas, faisant en sorte que toute conversation entre les deux peuples puisse se dérouler sans problème. Cependant nous savons fort bien de notre point de vue que le terme a une extension différente selon qu'il est utilisé par un habitant de l'une ou l'autre des planètes, puisqu'il ne désigne pas le même liquide. L'état psychologique d'un locuteur ne peut donc pas déterminer l'extension d'un terme. Selon Putnam cet exemple suggère qu'il y a une composante indexicale qui entre dans la détermination de l'extension. Soit un échantillon d'un liquide que nous pouvons pointer du doigt: nous appelons alors " eau " tout liquide de la même espèce que le liquide désigné. Les locuteurs de la Terre-Jumelle font de même, ce qui explique que les extensions des deux termes soient différentes. La spécification de la nature propre à ces espèces peut demander une quantité indéterminée de recherches théoriques et est donc en plus sujette à certaines modifications que peut entraîner le développement de la science. La recherche vise à découvrir les traits essentiels de l'espèce, c'est-à-dire les caractéristiques qu'elle possède qui sont responsables 
de ses propriétés et de son comportement. Le terme " eau " n'est donc pas synonyme avec la description de ses propriétés phénoménales, par lesquelles nous l'identifions couramment, comme l'affirmait la théorie traditionnelle. Dans les situations où l'espèce naturelle n'est pas directement accessible (et dans le cas des termes de grandeurs physiques) la composante indexicale qui détermine l'extension d'un terme mentionne les effets observables du référent: face à un résultat expérimental imprévu qu'il observe, un chercheur peut décider de nommer $E$ l'espèce (ou la grandeur) qui en est la cause. Il est possible qu'un autre chercheur soit, dans des circonstances différentes, témoin d'autres manifestations de cette même espèce (ou grandeur). L'espèce (ou la grandeur) est cependant définie comme la cause commune de ces effets, et non pas par leurs descriptions qui n'ont pour but que de l'identifier dans certaines circonstances.

Un deuxième argument décrit par Putnam fait appel à l'ignorance ou l'erreur du locuteur. La théorie de la signification traditionnelle semble pour sa part supposer que les locuteurs ont toujours une connaissance complète des caractéristiques propres des référents de leurs termes: puisque l'intension d'un terme doit contenir toutes les informations permettant d'identifier les objets auxquels il s'applique, le locuteur doit posséder ces informations pour être à même d'utiliser le terme. Certains exemples courants semblent pourtant démontrer le contraire: Putnam lui-même admet qu'il ne peut distinguer un orme d'un chêne. Il ne possède donc pas la connaissance requise par la théorie de la signification traditionnelle pour référer à ces arbres. L'intension qu'il associe au terme "chêne" est exactement la même que celle qu'il associe à «orme " mais nous disons pourtant toujours que les deux termes ont une extension différente dans son langage. Le cas de l'erreur n'est pas très différent: pour la plupart des gens la propriété " poisson » fait partie de l'intension de «baleine». Cette description est fausse mais cela n'empêche personne de référer aux baleines. En conséquence, dans un cas comme dans l'autre, l'état psychologique d'un locuteur ne permet pas de déterminer l'extension d'un terme. Selon Putnam ce deuxième type d'exemple prouve cette fois que l'extension d'un terme est déterminée en partie socialement. Il y a une division du travail linguistique: lorsque nous utilisons des termes scientifiques, nous empruntons aux experts qui connaissent les critères d'identification des objets la capacité d'y référer. Seuls les scientifiques experts ont une bonne connaissance des caractéristiques propres des espèces naturelles et sont en 
mesure de reconnaître les situations expérimentales causées par les mêmes grandeurs ou propriétés physiques.

Pour fixer une fois pour toutes l'extension de ces termes scientifiques ayant une composante indexicale et sociale, Putnam fait appel à la notion d'événement initial («introducing event »). Un événement initial est une situation nouvelle où le terme est pour la première fois utilisé par un expert ou simplement par un innovateur. Lorsque nous apprenons l'usage d'un de ces mots nous sommes reliés d'une certaine façon à ce baptême:

Once the term has been introduced into someone's vocabulary whether by an introducing event, or by his learning the word from someone who learned it via an introducing event, or by his learning the word by a chain of such transmissions to an introducing event, the referent in that person's idiolect is also fixed, even if no knowledge of that person has fixed it. (1975, p. 202)

C'est parce qu'elle requiert des relations de ce type que la théorie de Putnam est parfois appelée théorie causale de la référence.

La brisure du lien entre l'état psychologique et l'extension laisse Putnam avec deux possibilités: conserver l'idée que comprendre la signification d'un mot est la saisie de son intension et rejeter le principe selon lequel l'intension détermine l'extension ou abandonner la première des deux thèses pour faire de l'extension (ou de la référence) une partie intégrante de la signification d'un terme. Il choisit la seconde possibilité, qui permet de bloquer le chemin à la conception holiste de la signification. La signification d'un terme scientifique n'est alors plus dépendante $\mathrm{d}$ 'intensions, dans le sens où des différences dans l'extension des termes doivent nécessairement impliquer en plus des différences d'intensions: les différences en extension, déterminées indexicalement et socialement, sont ipso facto des différences de signification. Cette dernière n'est donc plus susceptible d'être dépendante de son rôle à l'intérieur d'une théorie.

La notion de signification prend chez Putnam la forme d'un vecteur à plusieurs composantes, dont la principale est l'extension. Dans la partie de la théorie qui est une théorie de la compréhension du langage, on retrouve les notions de marqueur sémantique et de stéréotype, deux autres composantes $\mathrm{du}$ vecteur de signification, qui s'apparentent à l'intension de la théorie traditionnelle. Les marqueurs sémantiques sont des catégories générales d'items, comme " animal " ou "liquide». 
Les stéréotypes sont des idées conventionnelles, pas nécessairement vraies, associées aux mots par le contact direct ou indirect des locuteurs avec leur extension, et sont habituellement des propriétés typiques possédées par les membres d'espèces naturelles qui nous permettent de les reconnaître dans des situations normales. Ils sont également suffisants pour communiquer ou pour transmettre la signification des mots dans la majorité des cas. Les stéréotypes sont utilisés par Putnam pour décrire la compétence linguistique des locuteurs mais ils ne fournissent bien sûr pas de critères nécessaires et suffisants pour déterminer l'extension des termes.

Comment la théorie de Putnam contribue-t-elle à régler le problème de l'incommensurabilité? En offrant un moyen pour dire que différentes théories peuvent être concernées par le même domaine d'objets. Dans l'optique de Putnam, à mesure que la science progresse, elle découvre certes des éléments nouveaux à propos d'objets et de leurs propriétés, mais si elle est parvenue à mettre la main sur une véritable espèce naturelle l'extension du terme la désignant reste inchangée à travers l'histoire malgré la possibilité de modifications théoriques ou l'amélioration des méthodes mises de l'avant pour l'identifier:

[...] (S)cientists use terms as if the associated criteria were not necessary and sufficient conditions, but rather approximately correct characterizations of some world of theory-independent entities, and they talk as if later theories in a mature science were, in general, better descriptions of the same entities that earlier theories referred to. (1975, p. 237)

Dans ce contexte il est possible de dire que Thompson et Bohr avaient tous deux une théorie sur les électrons, et qu'il $s^{\prime}$ agissait dans les deux cas des mêmes électrons que visent les théories d'aujourd'hui, même si aucune particule réelle ne correspond exactement à la description qu'ils en ont donnée. Pour ce faire il faut également appliquer une maxime que Putnam nomme le principe du bénéfice du doute ("The Principle of Benefit of Doubt »). Celle-ci consiste à dire qu'il faut accorder le bénéfice du doute à l'innovateur qui nomme pour la première fois un objet, ou à l'expert qui reprend le terme pour nommer le même objet, quant à l'identité des objets qu'il désigne. Étant donné sa place dans notre histoire scientifique, avec son évolution vers les théories actuelles, nous pouvons dire que Bohr, mis au courant de ces théories récentes, accepterait que l'on apporte des modifications aux descriptions qu'il a utilisées de façon à 
ce que l'on puisse confirmer qu'il référait à l'objet que la physique nomme maintenant électron. Ce principe doit toutefois être appliqué raisonnablement, pour que l'on puisse aussi rendre compte du cas des termes comme "éther " ou " phlogistique ", qui se sont avérés ne pas désigner d'espèce naturelle, ayant par conséquent une extension nulle. La théorie de Putnam démontre donc que les termes scientifiques $n^{\prime}$ ont pas besoin d'avoir la même signification - si par là on veut dire intension - pour que les théories soient comparables. Seule la continuité de la référence importe, et le principe du bénéfice du doute est justement une maxime qui permet de préserver la référence à travers les changements de théories.

\section{TROIS PROBLÈMES POUR LA THÉORIE DE PUTNAM}

Dans son excellent livre intitulé Representing and Intervening, Ian Hacking relate trois épisodes authentiques de l'histoire de la physique où des espèces naturelles furent pour la première fois découvertes ou nommées. Selon Hacking, si la théorie de Putnam nous libère du problème de l'incommensurabilité, elle n'en est pas moins imparfaite dans le détail parce qu'elle ne s'applique pas facilement aux trois exemples qu'il discute. Il présente ces trois exemples et les problèmes qu'ils posent sans les résoudre, comme un défi à relever: "Those who wish a theory of meaning for scientific terms will have to improve on Putnam ". (1983, p. 90) Notre intention est de relever le défi; mais il n'est pas certain que la théorie qui résultera de notre analyse ressemblera encore à la théorie originale.

\section{A. Problème pour l'aspect causal-historique de la théorie}

En 1936 un groupe de chercheurs menant des expériences sur les rayons cosmiques découvre que certaines des particules les constituant ne sont pas des électrons: elles n'obéissent pas à l'équation de perte en énergie que prévoit l'électrodynamique quantique. Ces nouvelles particules, dont la masse est moindre que celle du proton mais supérieure à celle de l'électron, sont alors baptisées du nom de méson. Pendant ce temps, Yukawa, s'interrogeant sur la nature des forces qui cimentent les noyaux atomiques, est amené à postuler l'existence d'une nouvelle particule, dont la masse est aussi située entre celle de l'électron et du proton. On supposa alors que les spéculations théoriques et les résultats expérimentaux pouvaient avoir un rapport entre eux et on conserva le nom donné par les expérimentateurs pour désigner la nouvelle particule. Des recherches subséquentes 
démontrèrent toutefois que le temps de vie de la particule détectée dans les rayons cosmiques ne correspondait pas aux exigences posées par la théorie de Yukawa. Une autre particule, également découverte dans les rayons cosmiques, s'avéra être la particule cherchée par Yukawa et fut nommée "mésonpi ». La particule de 1936, que l'on considérait maintenant comme étant complètement différente, fut alors rebaptisée "méson$\mathrm{mu}$ " d'abord, et "muon » ensuite. Dans cette histoire, le terme "méson» acquit donc une sorte d'intension, voulant dire "ce qui satisfait aux exigences de la théorie de Yukawa", et la particule de 1936 perdit son nom original lorsque l'on réalisa que cette intension ne correspondait plus à l'objet d'abord baptisé.

Cet exemple illustre les difficultés que peut entraîner l'association de la détermination de la référence et l'extension d'un terme avec des événements historiques. Entre le moment où la référence d'un terme est fixée par un événement initial dans une communauté donnée et son usage ultérieur par d'autres personnes plusieurs choses peuvent se produire, et il n'est pas certain qu'on puisse rendre compte de toutes ces possibilités en exigeant que tous les utilisateurs soient reliés à l'événement initial par un lien causal de type approprié, une notion notoirement obscure s'il en est une. Ainsi Fuller prétend que « it is doubtful that the sort of knowledge required by the causal theory of reference has ever existed " (1988, p. 84), voulant par là indiquer qu'il doute que les cultures aient le moyen de s'assurer que les actes de langage procèdent toujours de manière constante et systématique. Dans ce contexte il est possible que plusieurs usages différents du même terme se développent, même lorsqu'il est introduit de façon spécifique par un événement initial, faisant en sorte qu'il acquière une fonction référentielle complexe rendant inutile toute recherche d'un baptême quelconque.

Quoi qu'il en soit, nous voulons, même s'il y aurait beaucoup à dire, limiter la discussion sur ce sujet, et endosser la version non-historique de la théorie putnamienne proposée par NewtonSmith dans The Rationality of Science, qui a le mérite de permettre de multiples baptêmes, comme dans l'exemple donné par Hacking. Ceci est d'autant plus justifié que, comme l'a bien vu Elgin, "because science is a critical, self-reflexive discipline, the fact that terms appearing in two theories have a common etymological origin is inadequate to show that they have a common referent " (1983, p. 13), il semble bien que l'on puisse séparer l'aspect 
causal-historique du reste de la théorie, sans automatiquement réintroduire le problème de l'incommensurabilité. Nous avons vu que la stratégie adoptée par Putnam pour contrer la menace causée par l'incommensurabilité consiste en effet simplement à développer une notion de référence extra-théorique, en s'appuyant sur le principe du bénéfice du doute. Si l'idée d'une origine commune aux termes n'est d'aucun secours en ce sens, alors elle apparaît comme un appendice de la théorie et n'est pas essentielle à notre propos dans cet article.

La modification apportée par Newton-Smith est très simple et pourrait même être imputée à Putnam lorsqu'il n'est pas question d'événement initial dans ses écrits ${ }^{4}$. Newton-Smith propose de s'en remettre toujours aux experts de l'époque concernée pour la détermination (de façon indexicale) de l'extension des termes scientifiques. La théorie conserve alors tous les avantages qu'elle avait auparavant, tout en autorisant des changements dans la nomenclature et la possibilité de variations dans la référence des termes lorsqu'il y a des changements de théorie. Pour rendre compte de l'exemple que nous venons de discuter il suffit alors de dire que la particule de 1936 a été renommée, que la référence du terme «méson » a changé avec le développement de la théorie de Yukawa et que l'extension des noms des deux particules est maintenant déterminée par les critères d'identification actuels, sans égard à l'événement initial où le méson fut pour la première fois baptisé. L'extension de "méson" n'est alors aucunement liée à ce premier baptême.

\section{B. Problème pour la théorie de la signification}

À la suite du rejet par Lavoisier de la théorie du phlogistique, un autre traitement des problèmes entourant la substance chaleur était requis. C'est alors que les théories du calorique furent proposées et développées par Berthollet, Biot et Laplace. Laplace, notamment, fut capable, à l'intérieur de sa théorie du calorique, de prédire la vitesse du son dans l'air, un problème toujours insoluble dans le cadre de la physique newtonienne de son temps. Cependant, de notre point de vue, et déjà selon celui de Rumford à l'époque, la substance appelée "calorique", pas plus que celle nommée "phlogistique», n'existe tout simplement pas. Comme le phlogistique et le calorique ne sont pas des espèces naturelles, l'extension de ces termes doit donc être nulle dans les deux cas. 
Comment la théorie de Putnam peut-elle expliquer la transition du phlogistique au calorique? Nous savons qu'elle affirme que c'est principalement l'extension qui détermine la signification des termes scientifiques, s'opposant en cela à la théorie holiste qui défend plutôt la thèse que la signification de ces termes est fonction du rôle qu'ils jouent à l'intérieur de la théorie. Dans l'exemple qui nous intéresse, nous avons donc ironiquement le cas de deux groupes de chercheurs qui échangent sur le même sujet, c'est-à-dire, bien sûr, rien. Biot et Laplace étaient pourtant heureux de savoir que le calorique n'était pas semblable au phlogistique dont leurs prédécesseurs discutaient avec autant de conviction. La théorie de Putnam ne parvient pas à expliquer aisément pourquoi la signification de "calorique » peut être différente de celle de "phlogistique". Les stéréotypes ne sont pas d'un grand secours sur ce plan parce que ce ne sont pas eux qui fixent la signification des termes. Selon nous la leçon à tirer de cet exemple est que l'on ne peut considérer comme Putnam que l'extension est un élément du vecteur de la signification d'un terme. Une théorie de la signification des termes scientifiques doit s'appuyer sur d'autres bases, ce que fera celle que nous allons construire.

\section{Problème pour la théorie de la référence}

Au début du XIXe siècle, Lavoisier et Dalton s'entendaient sur les éléments principaux d'une théorie considérant les substances ayant un goût amer en solution aqueuse et changeant la couleur de certains indicateurs comme des acides. En 1923 cependant, deux nouvelles caractérisations des acides furent proposées: Lewis affirma qu'un acide est un membre $d$ 'une espèce naturelle qui peut former un lien chimique composé d'une paire d'électrons avec une base; Bronsted et Lowry prétendirent pour leur part que toute substance ayant tendance à perdre un proton est membre de l'espèce. Les deux définitions, qui ne sont pas équivalentes pour toutes les substances, font toujours l'objet de nombreuses discussions parmi les chimistes. On s'entend en général pour dire que la différence est essentiellement une question de nomenclature, sans véritable contenu scientifique. Dans ce contexte la question peut se poser: quelle était l'extension du terme "acide" avant 1923? Certainement pas celle des acides de Lewis ou de Bronsted-Lowry selon les théories actuelles. Peut-être l'intersection de ces deux espèces? Ou encore peutêtre simplement toutes les substances caractérisées comme telles avant 1923? La dernière solution semble la plus plausible mais 
il est alors presque certain que les acides d'avant 1923 n'étaient pas des espèces naturelles, ce qui pose un problème pour Putnam car celui-ci fait appel à cette notion pour fixer l'extension d'un terme.

À notre avis cet exemple montre que la théorie de Putnam doit supposer que les espèces naturelles sont des essences réelles, c'est-à-dire des entités possédant des propriétés essentielles, qui les déterminent au-delà de tout schème conceptuel, alors qu'elles ne sont plus généralement que des concepts, qui expriment les propriétés caractéristiques que notre schème conceptuel actuel attribue à des groupes d'objets pour les identifier. Putnam nous dit que la relation indexicale "de même nature que", qui fait référence sans la décrire à la propriété essentielle de l'espèce, est théorique: elle peut demander une somme indéterminée de recherches scientifiques. Il ajoute qu'elle est également révisable: elle est sujette à erreur, même dans les cas en apparence les plus certains, par exemple celui où des locuteurs terriens de 1750 auraient nommé "eau " un liquide qui est en réalité de l'XYZ, comme pourraient le confirmer leurs successeurs de 1950. Selon Putnam de tels exemples montrent bien que notre connaissance de la nature du liquide désigné a changé dans l'intervalle, mais pas que c'est le cas pour la signification du terme correspondant, qui reste la même puisqu'elle est basée sur son extension. D'autre part, Putnam croit aussi que sa théorie peut rendre compte des cas où une présumée espèce naturelle (comme le cas des acides avant 1923) n'a en fait pas de propriété essentielle. La science considère habituellement que les propriétés essentielles d'une espèce sont des propriétés structurelles mais il se peut très bien qu'un liquide n'ait pas de structure définie, parce qu'il s'agit d'un mélange, par exemple. Dans de telles situations ce sont les propriétés superficielles qui deviennent déterminantes, et les termes scientifiques prennent un autre sens:

It could have turned out that the bits of liquid we call " water " had no important common physical characteristics except the superficial ones. In that case the necessary and sufficient condition for being " water " would have been possession of sufficiently many of the superficial characteristics. (1975, p. 241)

La situation semble donc se présenter comme suit: l'extension d'un terme est déterminée en partie indexicalement lorsque le spécimen qui permet de la fixer a une propriété essentielle tandis qu'elle est déterminée par des propriétés caractéristiques 
- et donc par une intension - lorsque ce n'est pas le cas. Ceci pose un problème puisque la relation "de même nature que " est à la fois théorique et révisable et nous savons pas a priori si nous sommes oui ou non en présence d'une espèce ayant une propriété essentielle. Nous ne sommes donc pas en mesure de déterminer l'extension d'un terme scientifique tant que nous ne possédons pas cette connaissance. En conséquence, notre théorie de la référence des termes scientifiques varie avec les connaissances scientifiques!

Dans les exemples considérés par Putnam la composante indexicale est nécessaire parce que la référence peut être effectuée malgré une connaissance déficiente: un individu mentionne un terme désignant tous les membres d'une même espèce, sans toutefois pouvoir préciser selon quel critère ils sont membres de la même espèce. Putnam considère alors la possibilité que l'extension puisse être déterminée socialement, en s'appuyant sur des experts. Il est indubitable que nous utilisons parfois les mots de cette manière; mais il ne faut pas oublier que les experts dont nous dépendons alors doivent aussi déterminer l'extension de leurs termes. Il y a même des cas où le fait de $\mathrm{s}^{\prime}$ en remettre à un expert ne nous avance à rien dans la détermination de l'extension d'un terme. Un exemple donné par Elgin nous permet de comprendre. Il y a présentement deux critères, apparemment équivalents, qui fixent l'extension de "chien»: le code génétique et l'origine historique. Les biologistes ne parviennent pas à décider lequel de ces critères est le plus important, ce qui ne semble pas entraîner de conséquences néfastes. Supposons toutefois que l'on découvre un jour sur une planète lointaine des animaux possédant le code génétique de nos chiens mais qui, de toute évidence, ne sont pas de la même lignée. Il n'y a alors pas de réponse définitive à la question de savoir s'ils sont des chiens, ni même pour les experts puisque ceux-ci ne s'entendent pas. Et on ne peut avancer qu'un des deux groupes d'experts fait fausse route et n'identifie pas la propriété essentielle de l'espèce naturelle sans retomber sur le problème rencontré au paragraphe précédent. À notre avis il faut plutôt dire, comme Elgin:

[...] (I)t is equally true that we defer to experts in other fields to settle the application of their terms. We look to musicians to determine the extension of " cadenza ", to lawyers to determine that of " contract " and to players to determine that of " chukker ". There appears to be nothing that differentiates the relation of science to its vocabulary from the relation of these other fields 
to theirs. [...] There is thus no basis for saying that science discover essences, but other fields do not. Nor is there any point in saying that each field discover its appropriate essences. For once we grant that a particular group of specialists is authoritative in deciding the application of a term, nothing is added by saying that they do so by discovering the essences of their objects. (1983, p. 15)

Il n'y a ni vrai ni faux sur le plan sémantique selon Elgin, seulement des conventions, des décisions.

La théorie de Putnam repose donc sur l'idée que la science identifie les propriétés essentielles des espèces naturelles et exige que celles-ci aient une essence réelle ${ }^{5}$. Mais l'exemple de Hacking et la discussion montrent comment l'idée que l'on puisse trouver les propriétés essentielles des espèces naturelles n'est d'aucune utilité: elle ne pourrait servir que dans un futur utopique où la science nous présenterait des théories achevées (nous aurions alors connaissance de toutes les propriétés essentielles). Avant d'être des essences réelles, les espèces naturelles sont $d$ 'abord des concepts, des entités linguistiques qui forment les systèmes de classification des régularités que nous découvrons dans la nature, à la fois commodes d'un point de vue pratique et fondés d'un point de vue théorique, et qui évoluent, parfois radicalement, avec la science elle-même. Parmi la somme des propriétés associées à un temps donné aux espèces naturelles, il y a généralement une propriété caractéristique permettant de les définir par delà les autres, par l'autorité que lui confère la communauté scientifique. À cette différence près, la propriété caractéristique a le même statut que les stéréotypes, et permet donc de déterminer de façon précise (autant que possible) l'extension des termes d'espèces naturelles.

Mais si, comme nous le disons maintenant, seules les propriétés caractéristiques devraient intervenir au sein de la théorie de la référence des termes scientifiques, cela signifierait qu'il n'y a plus de composante indexicale qui entre dans la détermination de leur extension. Nous retrouvons à toutes fins pratiques la théorie de la signification traditionnelle, selon laquelle l'état psychologique et l'intension déterminent l'extension des termes scientifiques. Qu'advient-il alors des arguments de Putnam contre cette théorie? Comment peut-on expliquer, plus particulièrement, la fable de la Terre-Jumelle? Pour le savoir il nous faut introduire une distinction qui s'avérera capitale, celle entre référence et dénotation ${ }^{6}$. 


\section{RÉFÉRENCE ET DÉNOTATION}

La dénotation est une relation purement sémantique, abstraite de tout contexte d'énonciation, entre des termes linguistiques et des objets réels, qui ne dépend que de la signification attachée aux termes. La notion de vérité est impliquée dans cette relation: on associe des objets à un terme en vertu du fait qu'il est vrai qu'ils possèdent la propriété qu'il exprime. Au terme général " célibataire », signifiant "adulte non marié », on associe tous les adultes non mariés; au prédicat "analphabète ", signifiant "individu qui ne sait ni lire ni écrire", on associe tous les individus qui ne savent ni lire ni écrire. Le terme linguistique n'est pas simplement une étiquette regroupant sous un même nom un ensemble d'objets, comme le croient les nominalistes, car il n'y a en général aucun moyen de déterminer cet ensemble d'objets si ce n'est selon la propriété qu'ils ont en commun, telle que spécifiée par la signification du terme. La relation de dénotation fait intervenir l'intension et l'extension: l'intension est cette entité abstraite qui est exprimée par un terme, et par ce fait elle détermine l'extension, la classe des objets pour lesquels il est vrai de dire que les propriétés s'appliquent.

Par opposition, la référence, selon l'usage que nous voulons lui réserver à partir de maintenant, est une relation pragmatique entre des individus et des objets physiques dans un contexte donné. Elle se fait généralement par l'intermédiaire d'expressions linguistiques dans un acte de langage. Dans la référence ce n'est plus l'intension d'un terme mais l'intention d'un locuteur qui détermine quels objets sont désignés. En tant qu'acte elle $n^{\prime}$ est ni vraie ni fausse mais réussie ou non; il est donc inexact de parler de l'extension d'une référence. Par analogie avec la dénotation on peut utiliser la référence pour désigner une relation entre une expression linguistique et un objet $O$ : dire que l'expression réfère à $O$ est alors une façon abrégée de dire que les locuteurs réfèrent à $O$ en utilisant $l^{\prime}$ expression linguistique (on dira alors que $\mathrm{O}$ est le référent de l'expression). La référence est directe lorsque les objets sont pointés du doigt, les locuteurs utilisent alors des expressions indexicales, mais lorsque ceci est impossible les locuteurs sont contraints d'utiliser des expressions linguistiques descriptives. Dans la plupart des situations courantes, celles-ci dénotent les objets auxquels ils veulent référer, bien qu'il est possible que ce ne soit pas le cas. L'intension du terme qu'utilise un locuteur ne capture pas nécessairement son intention de référence, comme Donnellan, entre autres, l'a abondamment illustré. L'exemple 
de l'homme que l'on désigne par le fait qu'il boit un martini, mais qui boit en fait une eau minérale, est bien connu.

La distinction entre les deux notions nous permet de reconsidérer la fable de la Terre-Jumelle sous un angle nouveau. Lors de notre présentation de l'argument devant démontrer que l'état psychologique ne peut déterminer l'extension d'un terme nous avions assimilé l'état psychologique du locuteur à la saisie d'une intension. Un autre type d'état psychologique d'un locuteur pourrait être une intention de référence. Dans ce second cas, il est exact de dire avec Putnam que l'état psychologique ne détermine pas l'extension d'un terme: les locuteurs de la Terre et de la Terre-Jumelle de 1750, s'ils avaient eu l'occasion d'interagir, auraient tous eu l'intention de référer par le mot "eau " à ce qu'ils auraient cru être le même liquide, ne sachant pas que ce n'est en fait pas le cas. Ils auraient donc été dans le même état psychologique sans pourtant que cela suffise à déterminer la nature du référent. Si l'on considère toutefois que l'état psychologique dont il est question est la saisie d'une intension, la relation de dénotation, selon la définition que nous lui avons donnée, devrait faire en sorte que l'extension du terme soit immédiatement déterminée d'après le contenu de cette intension. Et si nous pouvons nous persuader que, bien que ce ne soit plus le cas à l'heure actuelle, l'intension du terme " eau » ait été la même pour tous les locuteurs en 1750, la possibilité que l'intension détermine l'extension demeure. Voici comment cela est possible à notre avis: avant la découverte de la composition chimique de $\mathrm{H}_{2} \mathrm{O}$ et $\mathrm{XYZ}$, cette propriété ne faisait pas partie de l'intension du terme "eau », qui désignait alors sur les deux planètes le liquide que l'on retrouve dans les lacs et les rivières, sans égard à sa composition chimique (l'intension du terme était alors quelque chose comme «le liquide que l'on trouve dans les lacs et les rivières "). Sur la Terre ce liquide est en fait de $\mathrm{l}^{\prime} \mathrm{H}_{2} \mathrm{O}$, et sur la Terre-Jumelle, de $l^{\prime} X Y Z$, mais cela aurait très bien pu être le contraire, ou encore un mélange $d^{\prime} \mathrm{H}_{2} \mathrm{O}$ et $d^{\prime} X Y Z$ sur les deux planètes. Ce n'est qu'avec le développement de la science que l'élément " composition chimique $=\mathrm{H}_{2} \mathrm{O}$ (ou XYZ)" fit son apparition au sein de l'intension du terme, et que la question de savoir si les deux liquides sont identiques de ce point de vue se posa. Auparavant, seuls les critères d'ordre pratique importaient. Dans cette nouvelle perspective, il suffit de dire que l'extension du terme " eau " a changé sur chaque planète depuis 1750 une tentative de résolution de l'énigme que Putnam a écartée 
d'emblée (1975, p. 224). Ce qui n'aurait effectivement pas changé sur une même planète $\mathrm{n}^{\prime}$ est pas l'extension du terme mais l'objet de référence des locuteurs.

C'est en se basant sur le sens prédominant d'un terme scientifique, celui qui a une composante indexicale, que Putnam affirme que l'extension du terme «eau » dans la fable de la Terre-Jumelle ne change pas sur une même planète. Putnam admet en effet que les termes scientifiques peuvent avoir plusieurs sens selon le contexte: nous désignons parfois un liquide par le nom "eau " même s'il contient des impuretés alors que cela peut être capital qu'il n'en contienne pas à un autre moment; de plus, notre utilisation habituelle du terme désigne un liquide mais en d'autres circonstances il est possible que seule l'espèce nous importe sans égard à son état physique (glace ou vapeur). Beaucoup d'autres exemples sont possibles, notamment le cas déjà considéré dans la section 3 . C, où il $n^{\prime} y$ a en fait pas d'espèce naturelle. Cependant, Putnam maintient, contrairement à ce que notre compte-rendu de la fable de la Terre-Jumelle exige, que le sens prédominant du terme ne fait pas intervenir (au niveau de son intension) la propriété caractéristique utilisée pour identifier les membres d'une espèce naturelle. Le sens prédominant du mot " eau », dit-il, contient l'idée d'une nature commune, mais pas la description de cette nature particulière $\left(\mathrm{H}_{2} \mathrm{O}\right.$ ou $\left.X Y Z\right)$. L'affirmation se base parfois sur le fait que, puisqu'il en existe plusieurs types différents, le test qui fixe l'extension du terme ne peut pas faire partie de sa signification (1975, p.151). Cet argument passe à côté de la question. Bien sûr plusieurs tests différents peuvent permettre de vérifier la nature d'un échantillon; leur rôle n'est pas de définir les termes mais toujours de déterminer si les échantillons possèdent ou non la propriété caractéristique. Un second argument - le principal - fait valoir que la signification du terme ne peut être synonyme avec la description de la propriété caractéristique (1975, pp. 141 sq.), comme en témoigne le fait qu'un énoncé comme "l'eau est $\mathrm{H}_{2} \mathrm{O}$ " n'est pas analytique, contrairement à l'exemple "un célibataire est un adulte non marié ». Ce nouvel argument énonce une vérité mais tire une fausse conclusion: les stéréotypes associés à " eau " ne sont pas non plus synonymes au terme, ce qui ne les empêche pas de faire partie de sa signification (selon la théorie de Putnam lui-même). En fait, stéréotypes et propriété caractéristique sont confondus dans un énoncé comme «l'eau est $\mathrm{H} 2 \mathrm{O}$ », et c'est ce qui fait que la question de son analyticité est ambiguë. Dans un premier 
sens l'énoncé n'est pas analytique mais est une hypothèse (ou découverte) empirique: " eau » a alors la signification du stéréotype courant "le liquide des lacs et des rivières", qui permet une première identification de l'extension mais autorise d'autres recherches. Dans un second sens l'énoncé est une définition et est donc analytique: " eau " a alors la signification que lui confère la propriété caractéristique qui détermine l'extension de façon précise. À la différence des propriétés caractéristiques de termes comme "célibataire" ("adulte non marié ") qui sont conventionnelles en ce qu'elles sont l'expression de rôles fonctionnels et sociaux, celles des termes d'espèces naturelles sont fonction des régularités que nous observons dans la nature et sont, par le fait même, sujettes à des modifications résultant de recherches empiriques. Mais cette dissemblance ne suffit pas pour justifier la nécessite de développer une théorie de la référence différente dans les deux cas.

Il est important toutefois de dissiper une confusion. L'idée que la propriété caractéristique peut être découverte par des recherches empiriques, jumelée à celle selon laquelle elle est partie intégrante de la signification des termes scientifiques, peut sembler impliquer que les significations sont des entités que l'on découvre empiriquement. En fait, elle implique plutôt que la signification de ces termes change car ce $n^{\prime}$ est pas la découverte de la propriété caractéristique comme telle qui constitue ipso facto un changement dans la signification du terme. Les faits sémantiques sont des entités historiques et conventionnelles: on obtient l'intension d'un terme par abstraction sur les usages référentiels que nous en faisons, que ceux-ci dépendent de faits sociaux ou empiriques, qu'ils soient établis par des linguistes ou des scientifiques. Certains usages sont peu répandus ou tout simplement pas encore institués. Il semble par exemple peu plausible de dire que la propriété caractéristique du tigre fait partie de la signification du terme parce que peu de gens sont à même de caractériser le code génétique du tigre (peutêtre aussi pour des raisons de commodité), mais l'idée ne semble pas si farfelue pour «eau». Lorsque la connaissance de la propriété caractéristique d'une espèce s'étend, elle devient partie intégrante de la signification du terme au même titre que les stéréotypes, à la différence près, comme nous l'avons mentionné, qu'elle a autorité pour la détermination de son extension. 
Même si nous considérons que ses arguments contre l'inclusion de la propriété caractéristique au sein de l'intension d'un terme sont insuffisants, il est important de chercher à comprendre pourquoi Putnam endosse la thèse de l'indexicalité, qui lui permet d'affirmer que l'extension du terme est toujours la même sur une même planète dans la fable de la Terre-Jumelle, créant ainsi un problème pour la théorie de la signification traditionnelle. À notre avis la confusion entre la dénotation et la référence est à l'arrière-plan de la discussion. En effet, la désignation d'une espèce naturelle par l'intermédiaire d'un spécimen est un acte de référence d'un locuteur et non pas, comme le requiert Putnam s'il veut pouvoir affirmer qu'elle permet la détermination de l'extension du terme correspondant, une exemplification de la relation de dénotation entre un terme et un groupe d'objets. Ceci est manifeste lorsque Putnam compare sa position à celle de Kripke:

It should be clear, however, that Kripke's doctrine that natural kind terms are rigid designators and our doctrine that they are indexical are but two ways to make the same point. We heartily endorse what Kripke says when he writes: "Let us suppose that we do fix the reference of a name by a description. Even if we do so, we do not then make the name synonymous with the description, but instead we use the name rigidly to refer to the objet so named, even in talking about counterfactual situations where the thing named would not satisfy the description in question. " (1975, p. 234)

La partie de la citation que nous avons mise en italique illustre hors de tout doute que Kripke et Putnam cherchent ici à caractériser une relation de référence. Celui que Putnam considère comme le sens prédominant d'un terme d'espèce naturelle est en fait un certain type d'acte référentiel. (De la même façon, les différents sens des termes d'espèces naturelles considérés par Putnam sont différents types d'actes de référence effectués par des locuteurs). Or, nous l'avons souligné, on ne peut déterminer l'extension d'un terme (par opposition au référent $d^{\prime}$ une expression) à partir d'une relation de référence. La question de la vérité dans l'utilisation d'un terme ne se pose que sur le plan de la dénotation; au niveau de l'acte de référence les mots ne sont que de simples étiquettes: l'utilisation de "eau" n'est pas plus vraie pour désigner l'objet qui est en fait $\mathrm{H}_{2} \mathrm{O}$ que pour celui qui en fait XYZ. Par abstraction sur l'acte de référence indexical dont parle Putnam, on peut considérer la possibilité d'une relation de dénotation entre un 
terme ayant une intension de la forme "de même nature que - ", possédant une composante indexicale, et un groupe d'objets, ce qui nous ramène toutefois au problème rencontré à la section 3. C.

Nous avons abondamment discouru sur le premier argument de Putnam contre la théorie de la signification traditionnelle, celui de la Terre-Jumelle, visant à introduire la composante indexicale dans la détermination de l'extension. Qu'en est-il maintenant de l'argument de l'erreur ou de l'ignorance, qui fait valoir la composante sociale dans la détermination de l'extension? Il illustre selon nous le fait que chaque personne n'associe pas nécessairement exactement la même intension à un terme. Si l'intension d' "orme " et de "chêne " est identique pour une personne on peut tout aussi bien prétendre que cette personne ne connaît pas bien la signification de ces termes, ou alors que l'intension qu'elle leur associe est la même que celle qu'elle associe, par exemple, à "variété d'arbre». Dans ce deuxième cas nous dirons simplement que l'extension de ces termes est la même pour cette personne, lorsqu'elle tente de les appliquer par elle-même à une situation particulière (ce qui ne l'empêche pas bien sûr de les utiliser aussi, dans certains cas, en s'appuyant sur des experts). Mais quoi qu'il en soit de notre explication de cette difficulté, il s'agit toujours d'un problème qui concerne la compétence linguistique des locuteurs d'une langue, et non pas la signification des mots de cette langue. Les significations de ces termes sont toujours disponibles en consultant un dictionnaire ou un ouvrage spécialisé et elles déterminent précisément, autant que possible - car l'exemple de "chien " montre que des situations nouvelles peuvent exiger que des décisions soient prises - l'extension que nous leur donnons collectivement.

La théorie de Putnam apparaît maintenant comme combinant des éléments d'une théorie sémantique et d'une théorie pragmatique. Du point de vue sémantique, il est exact de dire que l'extension est déterminée en partie socialement, au sens où ce sont les scientifiques qui confèrent son autorité à la propriété caractéristique d'une espèce naturelle, mais il est faux de dire qu'elle est déterminée en partie indexicalement, sauf si l'on accepte que les intensions peuvent avoir une composante indexicale, avec les problèmes que cela entraîne. Et, dans les deux cas, les composantes sociales et indexicales qui entrent dans la détermination de l'extension d'un terme ne vont pas à l'encontre de la thèse 
traditionnelle qui affirme que l'acte psychologique de saisie de l'intension d'un terme en détermine l'extension. Du point de vue pragmatique il est vrai que la référence peut s'effectuer indexicalement, sans l'utilisation de descriptions qualitatives. Cependant, cette indexicalité est limitée parce que l'intention de référence d'un locuteur, surtout lorsqu'il veut désigner un groupe d'objets, doit toujours comprendre un minimum de description, tout au moins pour préciser la catégorie générale de l'entité (substance ou événement par exemple) à laquelle il fait référence. En effet, dès que l'on veut désigner l'ensemble des objets de même nature qu'un spécimen, il nous faut préciser selon quel aspect. Car il y a un nombre indéterminé de façons selon lesquelles un objet que l'on désigne par un terme peut être semblable à un autre. Il ne suffit pas non plus de dire qu'il est membre d'une espèce naturelle, car les objets peuvent être membres de plusieurs espèces de ce type. Un animal peut être un membre de l'espèce cheval, probablement parce qu'il possède le code génétique commun à tous les chevaux, mais il est aussi un vertébré, en vertu du fait qu'il possède un squelette, ou un mammifère, parce qu'il a des mamelles ${ }^{7}$. En considérant l'exemple de l'eau, Putnam nous dit que seuls des objets de même substance sont impliqués. Cependant, comme le fait remarquer Papineau, ceci prend déjà pour acquis que la notion de substance a été explicitée, bien que l'histoire de la physique nous montre différents concepts de ce type (1979, pp.160-2). Même les notions de base comme "substance " doivent donc être précisées jusqu'à un certain point. D'autre part, étant donné qu'il s'agit d'un acte psycholinguistique individuel, il n'est pas du tout évident que la référence puisse être déterminée socialement, si l'on fait exception des cas où nous référons à quelque chose par l'intermédiaire d'une ou plusieurs personnes ${ }^{\theta}$. A la décharge de Putnam il faut dire qu'il n'est pas non plus clair que la théorie de la signification traditionnelle à laquelle il s'oppose sépare convenablement les questions pragmatiques des questions sémantiques. En fait il nous semble plutôt qu'elle cherche à donner une théorie purement sémantique de la référence des expressions linguistiques, ce qui est à nos yeux impossible. C'est d'ailleurs aussi l'opinion de Donnellan, qui écrivait en 1972:

It is the idea that only a backing of descrlptions identifying the referent by its fitting them (or some sufficient number of them) could serve to connect an object with a name that 1 question. On the positive side my view is that what we should substitute 
for the question "What is the referent?" is "What would the speaker be attributing the predicate to on this occasion? » $(1972$, pp. 376-7)

Dans ce passage on note que Donnellan substitue une question pragmatique à une question que l'on croyait d'abord sémantique.

\section{VERS UNE SÉMANTIQUE DES TERMES SCIENTIFIQUES}

Nous concevons la sémantique comme étant une partie de la linguistique. À l'intérieur de la linguistique, qui étudie les propriétés des expressions linguistiques en général (et qui ne doit pas être confondue avec les théories de la compétence linguistique, qui veulent expliquer ce que c'est pour un locuteur que de comprendre un langage), la sémantique est plus particulièrement concernée par celles qui touchent au problème de la signification. Elle n'est cependant pas une théorie de la signification, au sens philosophique du terme, qui cherche à répondre à la question «qu'est-ce que la signification?». La sémantique prend pour acquis que la signification existe et n'a pour but que le développement de modèles de fonctionnement du langage qui cherchent à rendre compte de nos intuitions linguistiques sur des catégories comme le caractère signifiant ( meaningfulness") des mots et des phrases, la synonymie, l'ambiguité ou l'analyticité ${ }^{9}$. Elle est aussi une discipline abstraite, comme les mathématiques, parce qu'elle n'a pas le souci de décrire la réalité psychologique des locuteurs. Pourtant, même si cela n'est pas nécessaire, les catégories de notre théorie sémantique peuvent avoir une origine psychologique. Considérons l'exemple de l'intension. En sémantique l'intension est une entité abstraite mais il est raisonnable de penser qu'elle est (en totalité ou en partie) dans une certaine correspondance avec la représentation mentale, qui est d'ordre psychologique. De la même façon, la relation logique entre l'intension et l'extension, qui est sémantique, n'est pas sans rapport avec le fait psychologique selon lequel nous sommes en mesure d'appliquer un terme lorsque l'on comprend la propriété qu'il exprime. Dire que la sémantique est abstraite ne signifie pas que nous en défendons une interprétation platonicienne car selon nous les entités abstraites n'ont pas d'autre lieu de résidence que le langage, compris au sens d'un produit historique de l'humanité.

Il n'est pas dans notre intention d'entrer dans les détails complexes d'une psychologie de la formation des représentations mentales ou d'une sémantique des théories physiques dans le 
cadre de cet article davantage concerné par les problèmes de la référence. Quelques indications sommaires suffiront. Afin d'être en mesure d'identifier les différents types de termes scientifiques, nous résumons ici les grandes lignes de notre conception de la structure de la théorie physique, le pragmatisme constructif, qui nous servira également à la prochaine section lorsque nous reconsidèrerons le problème de l'incommensurabilité. La théorie dans son ensemble est pour nous une perspective conceptuelle, un modèle théorique (au sens des physiciens), ni vrai ni faux mais plus ou moins applicable, permettant d'expliquer le comportement d'un domaine d'objets physiques et leurs propriétés. L'aspect constructif de notre position insiste sur le fait que les modèles théoriques sont des représentations de phénomènes physiques sous la forme de constructions abstraites, idéales et parfois fictives; l'aspect pragmatique indique que les modèles sont appliqués à certains secteurs du réel selon l'intention des scientifiques utilisant les théories. La théorie comporte trois constituants principaux:

(1) l'appareil analytique, qui comprend la structuration logicomathématique de la théorie (lois fondamentales, axiomes et postulats);

(2) les modèles, qui sont des systèmes d'objets abstraits, parfois idéaux ou fictifs, auxquels s'applique par définition l'appareil analytique;

(3) les assertions empiriques (ou lois phénoménologiques), qui sont des énoncés portant sur les objets du réel empirique que l'on peut obtenir en appliquant la théorie à une situation particulière du réel.

Par rapport à cette description, ce qui importe pour le propos de cet article est que la théorie soit une structure langagière à deux niveaux, non pas, comme dans l'empirisme logique, séparable en un vocabulaire théorique et un vocabulaire observationnel, mais plutôt constituée d'un langage mathématique, l'appareil analytique, qui régit le comportement des objets abstraits des modèles, par exemple les points matériels et solides géométriques de la mécanique classique, les rayons lumineux de l'optique classique, la fonction d'onde de la mécanique quantique ou même des substances comme le phlogistique et le calorique, et d'un langage physicaliste, système de classification en constante évolution à l'intérieur duquel on peut décrire le comportement d'objets réels, comme les particules matérielles ou les photons, dont l'existence a été prouvée expérimentalement, indépendamment des théories particulières qui les prennent 
comme objet, soit parce qu'ils sont directement perceptibles ou indirectement observables, par l'intermédiaire de leurs effets. En parallèle avec la distinction entre le langage mathématique et le langage physicaliste, nous distinguerons le vocabulaire abstrait - ainsi nommé pour le différencier du vocabulaire théorique de l'empirisme logique -, qui comprend les termes désignant les objets des modèles, entités linguistiques ou mathématiques qui ne sont que leurs propriétés, du vocabulaire physicaliste, composé des termes désignant les objets réels et leurs propriétés, et par le fait même de termes d'espèces naturelles et de grandeurs physiques. Dans notre optique les objets des modèles sont linguistiques non pas parce qu'ils sont compris comme de simples signes, mais au sens où ils n'ont pas d'existence en dehors du langage. Les termes du vocabulaire abstrait sont quant à eux aussi des entités linguistiques, des expressions qui cette fois ne sont que des étiquettes désignant un groupe de propriétés définissant un objet. Il y a donc deux types de termes principaux dans la théorie physique, les termes abstraits et les termes physicalistes.

Du point de vue psychologique, il nous semble clair que les représentations mentales correspondant à des parties de $l^{\prime}$ 'intension des termes physicalistes sont formées différemment de celles des termes abstraits. Les premières se construisent à partir des mécanismes psychologiques par lesquels l'esprit humain saisit les régularités de notre expérience du monde phénoménal. Le système conceptuel des objets du sens commun forme la base sur laquelle tout système théorique peut s'articuler. À mesure que l'expérimentation scientifique révèle des zones d'expériences inconnues, dont l'accès est rendu possible par la mise au point d'une instrumentation de plus en plus sophistiquée, le schème classificatoire des espèces naturelles et des grandeurs physiques se perfectionne, guidé par la théorie et contraint par la pratique. L'intension des termes abstraits ne peut cependant pas se constituer toujours de cette manière car, dans le cas des objets idéaux et fictifs, qui sont définis en partie - le cas des objets idéaux - ou en totalité - le cas des objets fictifs - par des propriétés idéales, il n'y a pas d'expérience phénoménale sur laquelle les mécanismes psychologiques peuvent prendre appui. La théorie de la formation des représentations mentales que l'on adoptera doit permettre la création de nouveaux concepts scientifiques à l'intérieur du langage. Rom Harré donne dans son livre Varieties of Realism un aperçu de la façon dont l'intension des termes abstraits peut être déterminée. La genèse de la 
signification des termes désignant des objets idéaux et fictifs, ou dont l'existence n'est qu'hypothétique, se base sur des analogies avec des expériences connues, s'élaborant à partir d'éléments de langage que nous possédons déjà. Harré suggère que la comparaison et la métaphore sont deux processus à considérer:

The tropes of simile and metaphor would seem to be the obvious candidates. Both are linguistic devices which create new meanings from within the resources of a lexical system and make no use of ostension to extralinguistic exemplars. In the case of simile, extralinguistic input is required for the litteral meaning of the term to be used, but its use as a simile does not depend upon a point-by-point comparison between the first and the second subject. Rather it creates that comparison. It invites the reader to look at the subject in such a way to emphasize certain aspects of it. Metaphor too extends the context of use of terms already having literal meaning, which may indeed have been based on extralinguistic exemplars, but it is used for just those occasion when we do not possess the linguistic resources to express what it is about the second subject that has struck us. It is not a comparison, but a catachresis. (1986, p. 208)

Par ces propos nous ne voulons que mettre en relief le contraste entre les deux types de vocabulaire que nous avons définis: la signification des termes physicalistes dépend directement ou indirectement (le cas des espèces non directement perceptibles) de notre expérience phénoménale du réel alors que celle des termes abstraits est fonction des modèles théoriques à l'intérieur desquels ils interviennent. Nous croyons que ceci implique que le holisme de la signification ne s'applique pas au vocabulaire physicaliste, mais il est clair qu'une théorie de la formation des représentations mentales serait requise pour appuyer les dires de ce paragraphe. Soulignons aussi que notre holisme au niveau de la signification des termes abstraits n'est pas du même type que celui des positivistes car il ne fait pas référence à des postulats de signification reliant les termes théoriques entre eux et reliant les termes théoriques aux termes observationnels ${ }^{10}$. En fait la distinction entre termes théoriques et observationnels ne joue aucun rôle dans le pragmatisme constructif.

La séparation des termes scientifiques en un vocabulaire abstrait et un vocabulaire physicaliste ne doit pas dissimuler la complexité de l'évolution sémantique de certains termes. "Photon ", un terme désignant aujourd'hui un groupe d'objets réels dont l'existence semble établie, et donc une espèce naturelle 
membre du vocabulaire physicaliste, a d'abord désigné un objet abstrait dans le modèle conçu par Einstein pour expliquer l'effet photoélectrique. À cette époque aucun objet de ce type n'avait encore été identifié. Le succès observationnel remporté par la théorie permit alors aux chercheurs scientifiques de mettre sur pied un programme expérimental pour en vérifier l'existence. Le passage d'un terme du vocabulaire abstrait au vocabulaire physicaliste est généralement accompagné d'un changement de signification: l'objet dont l'existence a été mise en évidence par l'expérimentation peut ne pas correspondre exactement à la description qui a orienté sa recherche.

Il est possible d'intégrer tous les éléments de notre discussion sur la signification des termes scientifiques jusqu'ici dans un vecteur à plusieurs composantes comme l'entendait Putnam. Nous avons toujours le marqueur sémantique, qui permet d'identifier la catégorie ontologique de base (objet, substance, espèce, grandeur ou mécanisme) et le type général de l'espèce naturelle (liquide, animal, etc...), , de la grandeur physique ou même de l'objet abstrait, les stéréotypes, qui demeurent les diverses idées associées au terme, et nous ajoutons maintenant la propriété caractéristique de l'espèce naturelle, de la grandeur ou de l'objet abstrait, telle que déterminée par les experts en la matière. L'ensemble de ces constituants forme l'intension du terme. Nous voyons que l'extension ne fait plus partie de ce vecteur - ce qui permet d'éviter les problèmes discutés à la section 3. B -, qu'il n'y a pas d'élément ayant une composante indexicale à l'intérieur de l'intension - ce qui élimine les difficultés rencontrées à la section 3. C - et que les espèces naturelles et les grandeurs physiques sont considérées comme des concepts, composées des différents éléments linguistiques que sont la propriété caractéristique, les stéréotypes et les marqueurs sémantiques. L'élément le plus important du point de vue de la science est bien sûr la propriété caractéristique; les stéréotypes ne font que rendre compte des usages courants du terme. Il y a également des cas où deux ou plusieurs propriétés caractéristiques se disputent l'approbation de la communauté scientifique et des cas où seuls des stéréotypes composent l'intension d'un terme, lorsqu'un phénomène est encore insuffisamment compris pour qu'on puisse en déterminer le trait caractéristique. Dans le contexte de cette théorie sémantique pour les termes scientifiques nous maintenons que l'intension d'un terme en détermine l'extension et que la signification des termes du vocabulaire abstrait est fonction du rôle qu'ils jouent à l'intérieur des théories, les 
deux thèses auxquelles s'était opposé Putnam. Avec ce qui apparaît comme un retour en arrière, la menace qui nous guette est évidemment le problème de l'incommensurabilité, qu'il est maintenant temps de reconsidérer.

\section{L'INCOMMENSURABILITE REVISITÉE}

La plupart des débats portant sur la question de l'incommensurabilité, inaugurés par Kuhn et Feyerabend, se déroulent toujours dans le cadre de la conception de la nature des théories scientifiques de l'empirisme logique ${ }^{11}$. Certes, Kuhn, Feyerabend et les membres de l'école historique sont parmi ceux qui rejettent la distinction entre vocabulaire théorique et observationnel, caractéristique de cette approche, de même que le caractère anhistorique de son analyse, mais aucun d'entre eux n'en propose réellement une critique approfondie sur le plan de la structure de la théorie ${ }^{12}$, comme l'approche sémantique et le pragmatisme constructif tentent de le faire. Afin d'évaluer la portée réelle des divers arguments, il faut à notre avis déplacer la problématique vers ces nouvelles perspectives. Nous allons ici nous limiter à examiner l'argument présenté à la section 1 dans le cadre du pragmatisme constructif. Nous avons jusqu'ici endossé intégralement les thèses (1) et (4) de l'argument. Les autres prémisses doivent maintenant être réévaluées; nous montrerons notamment que (5) est fausse pour les termes physicalistes et que (6) dissimule une confusion entre la dénotation et la référence.

Commençons par évaluer la conclusion (6). Dans l'optique de Kuhn et Feyerabend, puisque la signification d'un terme, qui dépend de la théorie, en fixe la référence, différentes théories peuvent référer à différents domaines du réel, même si elles utilisent les mêmes termes. Parce qu'il veut qu'il soit possible que des théories différentes utilisant les mêmes termes s'appliquent au même domaine d'objets, Putnam cherche à développer une théorie de la signification qui n'entraîne pas cette conséquence fâcheuse. C'est là, à notre avis, qu'il fait fausse route: ce n'est pas la théorie de la signification qui est en cause mais une méprise entre la dénotation et la référence. Kuhn et Feyerabend pensent comme si la relation sémantique de dénotation qui permet de déterminer l'extension des termes d'une théorie suffisait pour en déterminer également le domaine d'application. Notre approche pragmatique de la structure des théories nous fait voir que cela ne saurait être le cas: la théorie étant une perspective conceptuelle, plus ou moins applicable à certains 
secteurs du réel, qui peut être utilisée dans diverses circonstances, ne détermine pas par elle-même son domaine d'application. Les termes abstraits ne sont pas des descriptions d'entités qui existent comme telles aussitôt que la théorie remporte le succès observationnel, mais, au mieux, lorsqu'ils ne sont pas purement fictifs, des pistes pour la recherche d'entités réelles plus ou moins similaires. L'empirisme logique subordonnait ainsi la question du réalisme des entités de celle du réalisme des théories: il considérait la thèse réaliste selon laquelle la vérité empirique d'une théorie implique l'existence de ses référents; le pragmatisme constructif, par contre, inverse le rapport entre les deux questions: ce n'est que lorsque l'on a établi l'existence d'un objet physique que l'on peut considérer la vérité des assertions empiriques d'une théorie qui en décrit le comportement. Ce sont les scientifiques qui choisissent d'appliquer la théorie à un domaine donné et qui tentent d'établir la correspondance entre entités abstraites et réelles. Et la relation qui caractérise le rapport entre des locuteurs - ici des scientifiques - et un domaine d'objets en est une de référence.

La référence est certainement la notion dont Putnam a besoin pour établir la continuité du discours scientifique et mettre en défaut l'incommensurabilité: «The Principle of the Benefit of Doubt belongs to a methodology for saying what speakers refer to "; mais dans la même page il donne une description de la relation de dénotation: "Reference is a relation between words and the world; this is just a fancy way of saying that the extension of the relation 'refer to' is a class of ordered pairs of terms and things". (1975, p. 283) Le fait que Putnam cherche à caractériser une relation de référence est aussi évident lorsqu'il expose l'exemple suivant:

Suppose there are some particules which have virtually all the properties Bohr thought electrons have - call them "shmelectrons". But suppose that "shmelectrons " exist only in the other half of this universe. Shmelectrons satisfy the laws Bohr thought electrons satisfied; electrons and electricity do not. [...] Still there are no shmelectrons here; the particular phenomena Bohr was interested in are explained by the behavior of electrons, not shmelectrons (we suppose). (1975, p. 277)

Les shmelectrons de Putnam sont bien les particules que dénote le terme "électron" de Bohr - elles en possèdent toutes les caractéristiques - mais elles ne sont certainement pas celles auxquelles il réfère, celles avec lesquelles il croit entrer en relation par son dispositif expérimental. 
Kuhn et Feyerabend pensent que les questions de la détermination du domaine d'une théorie et de la continuité de l'histoire de la physique ne se posent qu'au niveau du langage, entre les termes de la théorie et leur dénotation. Mais la physique est avant tout la rencontre entre des chercheurs et des situations expérimentales. Putnam a raison d'insister pour dire que la référence est extra-théorique - nous dirons même extra-linguistique, voulant par là préciser qu'elle prend racine dans la pensée des locuteurs et qu'elle n'est ainsi pas relative à un langage donné - et que c'est ce fait qui permet de mettre en échec l'incommensurabilité. La détermination de la référence $n^{\prime}$ est en effet pas simplement une question de langage: nous avons noté le fait que la dénotation habituelle d'une expression et la référence du locuteur ne coincident pas dans tous les cas. L'histoire de la physique, au cours de laquelle des chercheurs tentent constamment de caractériser un domaine du réel, est nécessairement remplie d'exemples de ce type. L'intention de référence de deux chercheurs d'époques différentes peut être pratiquement la même - ils veulent référer au même domaine d'objets —, sans égard au langage qu'ils utilisent pour le faire. Mais en développant une théorie sémantique qui nous parle de l'extension des termes pour parvenir à prouver son point, Putnam ne fait qu'entretenir la confusion: la relation de dénotation demeure toujours linguistique et ne fournit pas le point d'attache qu'il cherche.

Le fait de poser la question de la continuité de la physique d'abord du point de vue de la référence (et donc de l'expérimentation) plutôt que $\mathrm{du}$ point de vue de la dénotation (et donc du langage) a certaines conséquences. On ne se demande plus, comme le fait $l^{\prime}$ instrumentaliste, si la science peut poursuivre ses activités de prédiction et de contrôle du comportement des objets du réel sans que ses termes théoriques dénotent ou, comme le fait le réaliste traditionnel, si le succès de la physique peut être rendu intelligible autrement qu'en supposant que ses théories sont de plus en plus vraies (ce qui implique que ses termes théoriques dénotent). Il faut maintenant se demander quels sont les objets que les scientifiques du passé sont effectivement parvenus à identifier et, à la suite de cela, s'ils énonçaient des vérités sur ces objets. Ceci peut comporter certaines difficultés auparavant insoupçonnées car le langage, notre seul mode d'accès à la science du passé, n'est peut-être pas le meilleur moyen pour s'acquitter de cette tâche, comme l'illustre l'écart entre la dénotation et la référence. Dans le 
cas d'un objet particulier, comme un objet céleste, ou d'une espèce naturelle facilement identifiable par ses effets, on peut en principe savoir s'il y a conservation de la référence. Les observations astronomiques sont consignées, et la théorie actuelle nous dit quelle était l'espèce naturelle qui causait l'effet qu'a observé un chercheur dans des circonstances précises. Mais si nous ne disposons que de la description de ce que les Grecs entendaient par "chrysos", la seule question à laquelle nous pouvons répondre est: « existe-t-il selon nous des objets correspondant à cette description?", et non pas: "les Grecs référaient-ils aussi à notre 'or' lorsqu'ils utilisaient ce terme? », car il est possible que leur description soit fausse et leur intention ne nous est pas accessible. Cet exemple nous rappelle encore une fois que l'on peut obtenir l'extension d'un terme quand nous en connaissons l'intension, mais que ceci n'est pas suffisant pour nous assurer de la continuité de la référence à travers la succession des théories.

On pourrait objecter qu'une fois que la continuité de la référence entre deux théories a été établie nous ne sommes pas pour autant directement libérés du problème de l'incommensurabilité, qui semble se poser à nouveau sur le plan de la dénotation: la thèse (5) dit bien que la notion d'extension - si ce n'est plus le cas pour la référence -, est intra-théorique. À notre avis, cependant, cette conclusion est fausse: dès que nous savons que des théories décrivent le même domaine d'objets, il suffit de choisir un système de classification unique pour comparer leur apport empirique respectif. À l'intérieur du pragmatisme constructif, ce système de classification est le langage physicaliste. Parce qu'il décrit le comportement d'objets réels dont l'existence a été établie indépendamment, le langage physicaliste est indépendant de théories particulières; il n'est que le lieu dans lequel leurs assertions empiriques sont exprimées. L'extension des termes qui le constituent contrairement à celle des termes théoriques de chaque théorie vraie dans le cadre de l'empirisme logique -, n'est donc pas relative à une théorie et ce même si rien n'assure que ce sera également le cas de l'extension des termes abstraits. Le pragmatisme constructif est donc en position pour affirmer, comme en (2), que l'intension des termes (du vocabulaire abstrait) varie en fonction de de la théorie dans laquelle ils sont définis et, comme en (3), que le même terme dans différentes théories n'a pas toujours la même intension, sans nécessairement conclure, comme en (5), que la notion d'extension est toujours intra- 
théorique, puisque ce n'est pas le cas pour les termes physicalistes. L'incommensurabilité sémantique ne suit de la combinaison de la théorie de la signification traditionnelle et de la conception holiste de la signification que si l'on subordonne, comme l'empirisme logique, et comme Kuhn et Feyerabend à sa suite, la question de l'existence des entités qui forment la population du réel à celle de la vérité des théories.

\section{CONCLUSION}

Comme nous l'avons mentionné au tout début, notre objectif principal dans cet article est de présenter les grandes lignes $\mathrm{d}^{\prime}$ une théorie de la référence des termes scientifiques des théories physiques. C'est la tâche qu'il nous reste, au terme de cet itinéraire qui nous a permis de réhabiliter la théorie de la signification traditionnelle (sur le plan sémantique) sans avoir à nous préoccuper de la question de l'incommensurabilité des théories physiques rivales. Nous arrivons en bout de parcours munis $d^{\prime}$ une distinction importante, celle entre la dénotation des termes et la référence des locuteurs.

Considérons le cas de la référence d'abord. Putnam nous semble avoir identifié les deux modes de référence principaux dont les scientifiques font usage: la référence déictique ou directe, pour les objets perceptibles, et la référence indirecte (par l'intermédiaire des effets), pour les objets en apparence inaccessibles. L'acte de référence directe peut être caractérisé par un énoncé de la forme générale "Ce $\mathrm{O}$ est un $\mathrm{C}$ " (où $\mathrm{O}$ est un objet et $C$ sa caractérisation en espèce naturelle), alors que celui de référence indirecte peut l'être par la forme générale « $C e$ qui est la cause de $E$ est un $C$ » (où $E$ est un effet et $C$ la caractérisation de sa cause en espèce naturelle ou en grandeur physique). La première composante de chacun des deux modes de référence $(O$ et $E$ ) relie le scientifique à un objet ou une situation particulière ${ }^{13}$. Dans le cas de la référence directe la réussite expérimentale de l'acte ne requiert que la capacité sensorielle d'identifier une figure sur un fond (ou un solide dans l'espace). Dans le cas de la référence indirecte la réussite de l'acte passe aussi par la conception d'hypothèses théoriques: après la perception de l'effet le chercheur doit tenter d'en donner une explication en termes de causes possibles. La réalisation expérimentale de l'acte peut toutefois être également qualifiée d'extra-théorique, en se basant sur un critère comme celui de Hacking, qui cherche à établir l'existence d'une entité indépendamment de l'hypothèse qui en fait mention, si les 
propriétés causales de l'entité en question peuvent servir pour l'investigation d'un autre domaine du réel: «By the time we can use the electron to manipulate other parts of nature in a systematic way, the electron has ceased to be something hypothetical, something inferred ». (1983, p. 262) La seconde composante des deux modes de référence tente de caractériser le type de l'objet ou de la situation en question, avec les ressources linguistiques disponibles (les significations en usage), ou par la création de nouvelles significations (par la comparaison ou la métaphore). $C^{\prime}$ est au niveau de cette seconde composante que la dénotation des termes utilisés peut ne pas coincider avec l'intention de référence, qui est habituellement celle de trouver les propriétés caractéristiques des espèces et des grandeurs.

La théorie de la dénotation des termes scientifiques doit respecter la distinction entre le vocabulaire abstrait et le vocabulaire physicaliste que notre analyse de la structure des théories physiques a mise en lumière. Une fois leur signification précisée (lorsque leur propriété caractéristique a été déterminée) les termes d'espèces naturelles dénotent les objets réels qui en satisfont la description. L'identification de ces objets peut évidemment demander une somme de recherche expérimentale indéterminée. Il est également possible que certains objets soient inclassifiables, révélant ainsi des lacunes du système de classification. Le cas des termes abstraits est différent, puisqu'ils n'ont pas toujours de dénotation. Les termes désignant les objets abstraits entièrement définis par des propriétés réelles dénotent les objets réels possédant toutes les propriétés correspondantes. Les termes désignant des objets idéaux et fictifs $n^{\prime}$ ont toutefois pas de dénotation, selon la définition donnée au début de la section 4, car ces objets, puisqu'ils sont définis en totalité ou en partie par des propriétés idéales, n'ont pas d'existence dans le réel empirique. $\mathrm{Au}$ lieu de prétendre qu'ils dénotent les objets abstraits des modèles, ce qui nous conduirait vers un platonisme que nous voulons éviter, nous voulons plutôt adapter à notre propos une suggestion de Elgin (1983, pp. 42-50) et dire que dans leurs discussions les scientifiques mentionnent les descriptions (d'objets abstraits) sans se préoccuper de leur dénotation. $\AA$ la différence de la dénotation, qui est une relation à deux composantes entre une expression linguistique et des objets réels, le prédicat de mention n'a qu'une seule composante et ne sert qu'à classifier les entités linguistiques que sont les objets abstraits. L'expression "gaz parfait", lorsqu'elle est mentionnée, permet de classer sous le terme toutes les descriptions de gaz 
parfaits, en vertu de la propriété caractéristique ou des stéréotypes associés, qui constituent l'intension du terme. Les termes de grandeurs physiques, dont l'intension exprime des propriétés réelles d'objets réels, sont aussi à ranger dans cette catégorie puisqu'en tant que telle une propriété est une entité abstraite. Les propriétés, réelles ou idéales, n'existent pas comme telles dans le réel (on $n^{\prime} y$ trouve que des exemplifications de propriétés); une propriété n'est qualifiée de réelle que par contraste avec une propriété idéale, parce qu'elle désigne une régularité empirique.

Il convient de mentionner en terminant que du point de vue de la recherche scientifique le fait que certains termes idéaux ou fictifs soient sans dénotation n'a pas pour les théories les conséquences dévastatrices auxquelles on pourrait penser. Lorsqu'ils discutent de gaz parfait ou de calorique les scientifiques ne font que tenter de caractériser des aspects de la réalité qui leur apparaissent importants d'un point de vue théorique; ils ne se critiquent pas entre eux sur la base du fait que la dénotation des termes qu'ils utilisent est nulle (ce que tout le monde sait) mais sur les éléments qui devraient ou non être inclus dans la description. Du point de vue théorique, l'intérêt de la discussion réside dans la description idéale ou fictive elle-même.

Département de philosophie

Université de Montréal

\section{NOTES}

1. L'article paraîtra dans Dialogue (voir la bibliographie).

2. L'argument que nous allons exposer n'est présenté explicitement ni par Kuhn ni par Feyerabend mais c'est ainsi qu'il est discuté par Putnam.

3. Newton-Smith, 1981, pp. 151-6 et Papineau, 1979, chap. 1, 2.

4. Dans un ouvrage plus récent, il semble d'ailleurs que Putnam luimême rejette cet aspect de la théorie. Il nomme maintenant sa théorie "la nouvelle théorie de la référence » (1983, p. 73).

5. Dans un article plus récent Putnam prétend que son essentialisme se limite aux intentions des locuteurs: «For what I have said is that it has long been our intention that a liquid should count as 'water' only it has the same composition as the paradigm exemples of water. [...] (T)he essence of water in this sense is the product of our use of the word, the kind of referential intentions we have. " (1983, pp. 220-1) Dans la section 4 vous verrons que, bien que ce 
transfert sur le plan de la pragmatique soit légitime, la détermination de l'extension d'un terme est sémantique. Putnam peut dire qu'une composante indexicale entre dans les actes de référence des scientifiques mais il ne peut affirmer que l'extension d'un terme est déterminée indexicalement.

6. La distinction que nous introduisons entre dénotation et référence se rapproche beaucoup de celle que fait Katz entre les notions de "type reference" - selon laquelle l'intension d'une expression linguistique permet à elle seule d'en déterminer le référent - et de " token reference " - selon laquelle les intentions du locuteur sont aussi à considérer (1981, p. 147). Mis à part le fait qu'il s'agit d'une relation entre des expressions linguistiques et des objets (et qu'il ne le considère donc pas comme pragmatique), le second type de référence de Katz semble correspondre assez fidèlement avec ce que nous appelons la référence. Le parallèle est moins marqué entre la "type reference " et la dénotation dans la mesure où Katz défend une conception platonicienne de la sémantique: les intensions des termes sont pour lui des universaux qui existent indépendamment de l'esprit humain (ibid, p. 172). Pour nous les intensions sont des abstractions prélévées sur la référence.

7. Devitt \& Sterelny, 1987, p. 73.

8. Devitt \& Sterelny, 1987, pp. 49-51. Ceci ne semble toutefois pas être d'un intérêt particulier pour la science.

9. Katz, 1981, pp. 119-24.

10. Il est bien question ici du holisme néo-positiviste basé sur la notion de postulats de signification et non pas du holisme sémantique, quinien, qui résulte de la combinaison de la thèse épistémologique duhémienne et de la théorie vérificationniste de la signification.

11. C'est le cas pour la majorité des travaux de l'école historique (Laudan, 1986, p. 146).

12. C'est également l'opinion de Laudan (ibid., pp. 157-8).

13. Harré présente un traitement similaire, distinguant le mode d'attribution DC (pour la référence directe) du mode d'attribution IP (pour la référence indirecte) (1986, chap. 4). 


\section{BIBLIOGRAPHIE}

BOURNEUF, ÉRIC, "La structure de la théorie physique et son contenu empirique. Le problème des idéalisations et des fictions » (à paraître dans Dialogue).

DEVITT, MICHAEL \& STERELNY, KIM, Language and Reality, Oxford, Basil Blackwell, 1987.

DONNELLAN, KEITH, "Proper Names and Identifying Descriptions". Dans Donald Davidson \& Gilbert Harman, dir., Semantics of Natural Language, Dordrecht, Reidel, 1972. pp. 356-79.

ELGIN, CATHERINE, With Reference to Reference, Indianapolis, Hackett Publishing Company, 1983.

FULLER, STEVE, Social Epistemology, Bloomington, Indiana University Press, 1988.

HACKING, IAN, Representing and Intervening, Cambridge, Cambridge University Press, 1983.

HARRE, ROM, Varieties of Realism, Oxford, Basil Blackwell, 1986.

KATZ, JERROLD, Language and Other Abstract Objects, Totowa, N. J., Rowman \& Littlefield, 1981.

LAUDAN, LARRY, dir., "Scientific Change: Philosophical Models and Historical Research", Synthèse, vol. 69 (1986), pp. 141223.

NEWTON-SMTH, WILLIAM, The Rationality of Science, London, Routledge \& Kegan Paul, 1981.

PAPINEAU, DAVID, Theory and Meaning, Oxford, Clarendon Press, 1979 .

PUTNAM, HILARY, Mind, Language and Reality, Cambridge, Cambridge University Press, 1975.

PUTNAM, HILARY, Meaning and the Moral Scicnces, London, Routledge \& Regan Paul, 1978.

PUTNAM, HILARY, Realism and Reason, Cambridge, Cambridge University Press, 1983.

SCHWARTZ, STEPHEN, dir., Naming, Necessity and Natural Kinds, London, Cornell University Press, 1977. 